\title{
Georges Bataille oder die Enttabuisierung des Verdrängten
}

Es ist mit Sicherheit lohnenswert, sich zunächst mit einigen Biographemen des französischen Philosophen, Romanciers und Literaturtheoretikers Georges Bataille auseinanderzusetzen. Er wurde am 10. September 1897 in Billon im Département Puy-de-Dôme geboren und starb am 9. Juli 1962 in Paris. Sein Gesamtwerk mit zahlreichen Essays ist überaus facettenreich und erstreckt sich auf die unterschiedlichsten Gebiete und Disziplinen, von Politik, Soziologie und Ökonomie über Archäologie, Ethnologie und Religion bis hin zu Literatur, Kunstgeschichte und Philosophie. In den fünfziger Jahren bezeichnete ihn kein Geringerer als Martin Heidegger einmal als den besten denkenden Kopf Frankreichs.

In jungen Jahren stand Bataille dem Kreis der Surrealisten um André Breton und Louis Aragon recht nahe, ging gegenüber der Bewegung aber zunehmend auf Distanz, da er deren marxistische Orientierung nicht teilte. Sehr persönliche Auseinandersetzungen mit André Breton schlossen sich an. Bataille ging mehrfach in der von ihm geleiteten Zeitschrift Documents gegen den Surrealismus vor; Breton revanchierte sich mit Attacken im Zweiten Surrealistischen Manifest. Viele der von Breton ausgeschlossenen Surrealisten wie Leiris, Prévert, Queneau oder Vitrac scharen sich um Georges Bataille. Breton sah in ihm lange einen missliebigen Konkurrenten um die Macht im literarischen Feld. Erst spät kommt es zwischen beiden zu einer Versöhnung im Zeichen des überall in Europa siegreichen Faschismus, gegen den Bataille mobil machte.

Bataille entstammte einer wohlhabenden Bauernfamilie; sein Vater war an Syphilis erkrankt und bereits blind, als Georges auf die Welt kam. Seine Mutter litt unter Depressionen und war latent suizidgefährdet. Der junge Bataille half mit, den blinden Vater zu pflegen. Nach dem frühen Umzug der Familie nach Reims und der Schulzeit der Söhne kam man in die Wirren des Ersten Weltkriegs, in dessen Verlauf Reims unter schweren Beschuss geriet. Georges schloss sich mit seiner Mutter dem Flüchtlingsstrom an - sein älterer Bruder war an der Front -, doch ließ man den todkranken, nicht selten tobenden Vater in der Stadt zurück, da er den Strapazen der Flucht nicht mehr gewachsen zu sein schien und später im November 1915 verstarb. Bataille empfand die Flucht als ein Im-Stich-Lassen. Die Krankheit des Vaters und speziell dessen Blindheit traumatisierten Bataille: Die Thematik des Auges blieb für ihn lebenslang autobiographisch besetzt.

Neben seinem Romanschaffen entfaltete Georges Bataille ein umfangreiches literarisches, literaturtheoretisches und philosophisches Werk, dessen Einfluss auf den französischen Existenzialismus, den Strukturalismus und Poststrukturalismus kaum überschätzt werden kann. Georges Bataille schrieb unaufhörlich, 


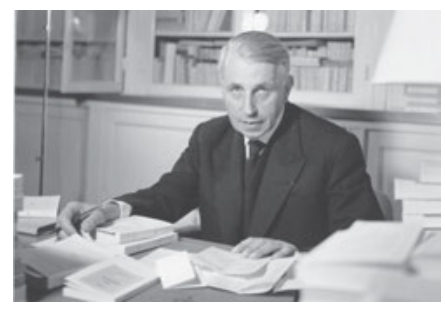

Abb. 84: Georges Bataille (Billom, Département Puy-de-Dôme, 1897 - Paris, 1962).

veröffentlichte Artikel um Artikel, Buch um Buch mit ungeheurer Energie. Seine gegen den Vater gerichtete Lebenseinstellung schlug sich in einer tiefen Suche nach dem Glauben nieder: Das Bekenntnis zum Katholizismus gab dieser Suche Ausdruck. Selbst nachdem sich der französische Philosoph von der Kirche wieder abgewandt hatte, blieb er doch mit zahlreichen seiner Publikationen der Religion und ihrer Geschichte verbunden. Früh wurde er von körperlichen Gebrechen geplagt: Während seiner militärischen Grundausbildung erkrankte er erstmals an Tuberkulose und kam in die Auvergne zu seinen Großeltern, litt sein Leben lang aber unter einer Immunschwäche seines im Übrigen durch starken Alkoholkonsum geschwächten Körpers.

Georges Bataille übte sich früh in Arbeit und Gebet, studierte am Priesterseminar in Saint-Flour, doch sein Wunsch, Priester zu werden, blieb unerfüllt. Er schlug nun eine Laufbahn als Bibliothekar ein, die er an der französischen Hochschule für Spanische Studien, der späteren Casa Velázquez in Madrid, als Archivar fortsetzte und brillant abschloss. Doch die spanische Hauptstadt bot ihm noch ein anderes Schauspiel: Am 22. Mai 1922 befand er sich in der Arena von Madrid und wohnte dem Tod des jungen Torero Manuel Granero bei, dessen Auge und Schädel von einem Stierhorn durchstoßen wurden. Auf die literarischen Folgen werden wir gleich $\mathrm{zu}$ sprechen kommen. Er arbeitete später zeitweise an der Nationalbibliothek zu Paris, in deren Dienst er gegen Ende seines Lebens schwer erkrankt zurückkehrte. Die Tuberkulose ließ ihn nicht gänzlich los; 1953 aber verschlimmerte sich sein Gesundheitszustand zunehmend: Bei ihm wurde Arteriosklerose im Hirnbereich diagnostiziert. Diese äußerte sich bei ihm bis zu seinem Tod in Form heftiger Kopfschmerzen und zunehmenden Gedächtnisverlusts, was ihn freilich nicht daran hinderte, bis zu seinem Lebensende unermüdlich Bücher zu schreiben und Zeitschriften herauszugeben.

In seinem philosophischen Denken, das am Paradox ausgerichtet war, arbeitete er dem Systemdenken Hegels entgegen und orientierte sich stark an Friedrich Nietzsche: Bereits 1922 begeisterte ihn die Lektüre von Jenseits von Gut und Böse. 1927 und 1928 verfasste und veröffentlichte er unter einem Pseudonym - vielleicht aus Rücksicht gegenüber seiner ersten Frau, die er 1928 heiratete - seine Histoire de l'Eil, mit der wir uns näher auseinandersetzen wollen. Im 
September 1933 publizierte er in zwei Teilen seine Überlegungen zu La structure psychologique du fascisme, eine bahnbrechende Arbeit über den Faschismus, die bis heute ihre Gültigkeit nicht verloren hat. Die literarische Philosophie Nietzsches prägte viele seiner Schriften und ist fast allgegenwärtig etwa in seiner subjektphilosophischen Arbeit L'expérience intérieure (1943), in der er sich wie in vielen anderen Arbeiten an nietzscheanischen Vorstellungen orientierte. Lektüren Sigmund Freuds schlossen sich an. Bataille unterzog sich einer später abgebrochenen, aber für ihn sehr wichtigen Psychoanalyse, die ihm Aufschluss über sich selbst gab.

In L'érotisme, das 1951 entstand, ging er von einem wechselseitigen Bedingungsverhältnis von Sexualität, gesellschaftlichen Sanktionen und Todeserfahrung aus, wobei er durch die Engführung von Eros und Thanatos wichtige Einsichten gewann, welche nicht zuletzt auf die Entwicklung der französischen Psychoanalyse durch Jacques Lacan - mit dem seine erste Frau Sylvia seit 1938 zusammenlebte - großen Einfluss nahm. Unermüdlich publizierte er zu Nietzsche und beschäftigte sich zunehmend mit dem Marquis de Sade: Seine Vorworte zu Ausgaben Sades setzten Maßstäbe und wurden berühmt. Gerade seine ökonomischen Vorstellungen psychischer Verausgabung und sein Zusammenspiel von Tabu, Grenzverletzung und Verbot beziehungsweise Bestrafung wirkten grundlegend auf Psychologie, Kulturtheorie und Philosophie sowie auf Denker von Michel Foucault über Julia Kristeva bis Roland Barthes.

Kommen wir nun zu jenem Text, den ich mit Ihnen zusammen anhand einiger ausgewählter Passagen erarbeiten möchte. Es handelt sich um Georges Batailles Histoire de l'EFil, eine Geschichte des Auges, die wir nur ungenügend klassifizieren würden, rechneten wir sie schlicht der erotischen Literatur zu. Es geht vielmehr, wie wir gleich sehen werden, um einen Text, der nicht frei ist von autobiographischen Elementen, der aber die erotische Dimension so weit radikalisiert, dass es sich im Grunde um einen „texte-limite“, um einen Grenztext handelt. Ich werde daher auch keineswegs alle verschiedenen Varianten von Grenzen im Rahmen dieser Vorlesung vorstellen können, sondern möchte es bei einigen aus meiner Sicht signifikanten Impressionen belassen, welche vor dem Hintergrund unserer Avantgarde-Diskussionen bedeutungsvoll sind.

Georges Bataille veröffentlichte diese Geschichte in eben jenem Jahr 1928, in dem auch Bretons Nadja erschien, einem Schlüsseljahr also der historischen Avantgarden im Allgemeinen und des Surrealismus im Besonderen. Es wäre freilich nicht ganz zutreffend, Georges Bataille dem französischen Surrealismus vollumfänglich zuzurechnen, denn die Beziehungen zu Breton waren - wie bereits betont - von Beginn an überaus gespannt. André Breton sah in Bataille einen von sexuellen Obsessionen Besessenen, und in seinem Second Manifeste du Surréalisme hagelte es gar persönliche Angriffe der schlimmsten Art gegen Bataille. 
Letzterer hatte freilich das Seine zur wechselseitigen Zuspitzung der beiderseitig unerfreulichen Beziehung geleistet.

Georges Bataille hatte auch eine Vielzahl von Freunden, darunter etwa André Masson, die dem Breton'schen Surrealismus nahe standen und verfolgte darüber hinaus, zumindest aus heutiger Sicht, Ziele und Vorhaben, die ohne den Kontext des Surrealismus oder insgesamt der historischen Avantgarden kaum vorstellbar gewesen wären. Während sich Breton aber einerseits dem Traum und dem Unbewussten, andererseits der Politik und der literarischen Parteibildung zuwandte, entwickelten sich die Interessen Batailles sehr viel stärker in Hinblick auf die körperliche Dimension menschlichen Erlebens. Dabei war es ihm um dieses Erleben in seiner Verbindung zum Spirituellen und Transzendenten einerseits zu tun und andererseits um eine radikale Grenzüberschreitung individueller Art, ohne dass dabei eine feste Gruppenbildung und Führerschaft miteingeschlossen oder auch nur mitgedacht gewesen wären. Denn Bataille ging es weder um die Leitung irgendeiner avantgardistischen Bewegung noch um eine wie auch immer geartete Konkurrenz zu Breton als Führungsfigur des französischen Surrealismus.

Doch trotz dieses Kontexts der historischen Avantgarde, durch den das Lesepublikum - und auch die Zensur - schon an vieles gewöhnt waren, konnte Georges Bataille seine Geschichte des Auges, der zu den frühen seiner Feder gehört, nur unter einem Pseudonym veröffentlichen. Bataille war gerade frisch verheiratet und zählte als 1897 Geborener zu jener Altersgruppe der über Dreißigjährigen, denen man - einem alten 68er-Spruch folgend - nicht mehr trauen sollte. Bataille, der in seinem Leben eine Vielzahl höchst kreativer Pseudonyme benutzte, wählte für seine Histoire de l'Fil „Lord Auch“, was man durchaus „Osch“ aussprechen sollte, denn hinter ihm verbergen sich die „chiottes“, also das Scheißhaus, das

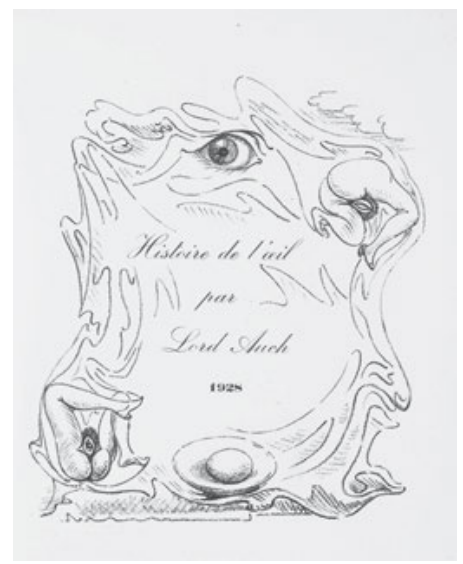

Abb. 85: Lithografie von André Masson in Georges Batailles Buch Histoire de l'CFil, 1928. 


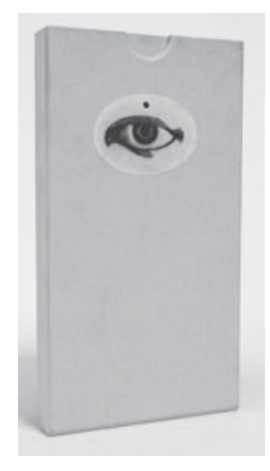

Abb. 86: Rosafarbener Umschlag des Pauvert-Verlages für Georges Batailles Histoire de l'Eil, Vignette von Pierre Faucheux, 1967.

(wie Bataille selbst anmerkte) mit dem englischen Namen Gottes in Verbindung gebracht wird. Sie sehen: Wie im Surrealismus Breton'scher Prägung allgemein findet sich auch bei diesem Denker und Philosophen im Besonderen ein blasphemischer Aspekt, der freilich bei Bataille aus einer ganz anders gearteten und zeitweise am Katholizismus orientierten Sinnsuche hervorgeht.

Es handelt sich bei der Histoire de l'Eil um einen Prosatext, der in vierzehn kleine, jeweils mit einem Titel überschriebene Kapitel eingeteilt ist, denen dann die „Réminiscences“ sowie ein kurzer Plan für eine Fortsetzung der Geschichte beigegeben sind. Das Incipit des relativ kurzen Textes weist eine „entrée en matière“ auf, die wir als ein Eintritt in medias res bezeichnen könnten. Denn in der Tat wird sofort die Grundkonstellation des gesamten Textes aufgezeigt. Ich gebe im Folgenden den Beginn des ersten Kapitels nur unwesentlich verkürzt wieder:

Ich wurde alleine erzogen und war, soweit ich mich daran erinnern kann, angsterfüllt mit Blick auf die sexuellen Sachen. Ich war fast sechzehn Jahre alt, als ich ein Mädchen meines Alters traf. Simone, am Strand von X ... Da es zwischen unseren Familien einen entfernten Verwandtschaftsbezug gab, wurden unsere Beziehungen dadurch beschleunigt. Drei Tage, nachdem wir uns kennengelernt hatten, waren Simone und ich allein in ihrer Villa. Sie hatte eine schwarze Schürze an und trug einen gestärkten Kragen. Ich begann zu vermuten, dass sie meine Angst teilte, die an jenem Tage umso stärker war, als sie nackt unter ihrer Schürze schien. [...]

„Die Teller sind dafür gemacht, sich hineinzusetzen“, sagte Simone. „Wettest Du? Ich setz’ mich auf den Teller.“

„Ich wette, dass Du das nicht wagen wirst“, antwortete ich atemlos.

Es war heiß. Simone stellte den Teller auf eine kleine Bank, installierte sich vor mir, setzte sich, ohne mich aus den Augen zu lassen, und tauchte ihren Po in die Milch. Ich blieb einige Zeit unbeweglich, das Blut war mir in den Kopf gestiegen und ich zitterte, während sie zusah, wie meine Rute die Hose spannte. Ich setzte mich zu ihren Füßen. ${ }^{1}$

1 Bataille, Georges: Histoire de l'Eil. In (ders.): Euvres complètes I: Premiers écrits, 1922-1940. Paris: Gallimard 1970, S. 571. 
Wir haben es hier mit einer Schlüsselszene der gesamten Erzählung oder vielleicht besser dieser Novelle zu tun: Denn es handelt sich in der Tat um eine relativ kurze, abgeschlossene, an einem einzigen Handlungsstrang orientierte Geschichte einer unerhörten Begebenheit - und dies entspricht genau der Gattungsdefinition einer Novelle. Die beiden zentralen Protagonisten sind ein Ich-Erzähler, der männlich und vielleicht knapp sechzehn Jahre alt ist sowie eine weibliche Figur namens Simone, die sich irgendwo am Strand zufällig kennenlernten. Beide sind weitläufig miteinander verwandt und verfügen noch nicht über sexuelle Erfahrungen, aber sehr wohl über eine große ,angoisse“ und Ungeduld, diese endlich zu sammeln. Das also ist die Ausgangssituation unserer Novelle!

Die Aktivität in dieser Szene geht von der weiblichen Figur aus, die sehr schnell schon als obskures Objekt der Begierde des Ich-Erzählers erscheint. Sie ist es, die die Initiative ergreift und spielt mit den beiden französischen Lexemen von „assiette“ und „asseoir“, ein Wortspiel, das im Deutschen nicht wiederzugeben ist und überdies englischsprachige Resonanzen aufruft. In dieser Passage lässt sich leicht eine gewisse Fetischisierung des weiblichen Körpers belegen, wobei an einer Stelle, die ich oben nicht zitiert habe, noch keine Trennung zwischen weiblichem Geschlechtsteil und Anus erfolgt, werden beide doch unter den Begriff „cul“ (oder engl. ,ass“) gepackt.

Gleichwohl ist es die bedeckte, die tabuisierte erogene Zone des weiblichen Körpers, die in dieser Szene im Mittelpunkt steht und zum Gegenstand der männlichen wie der weiblichen Erregung wird. Denn ihre Genitalien werden von Simone nicht etwa nur ausgestellt, exponiert oder verhüllt, sondern gleichsam verstellt, einem anderen Zwecke zugeführt: Sie werden auf einem Teller in Milch gebadet, was zur Erregung beider führt. Denn wir haben es ganz offenkundig mit zwei französischen Minderjährigen zu tun, die die Zeit, in der sie alleine in einer Villa sind, gemeinsam ausnutzen, um in einem unausgesprochenen Einverständnis ihre sexuellen Ängste zu überwinden.

Die sich aus dieser Konfiguration ergebende abhängige und zunächst inaktive Position des Männlichen wird gleich in dieser Eingangsszene verräumlicht dargestellt, erscheint der männliche Erzähler nun doch mit hochrotem Kopf zu Füßen des weiblichen Geschlechts sitzend, das er in dieser Inszenierung zum ersten Mal zu Gesicht bekommt. Simone lässt - und dies ist ein wichtiges und sehr aussagekräftiges Detail - den Ich-Erzähler nicht aus den Augen: Die Augen werden so nicht nur auf Ebene des Sehens und Gesehen-Werdens - und damit in einem Kontext des Voyeurismus -, sondern auch als Fixierungspunkte zu wichtigen Trägern der Handlung. Nur am Rande verweise ich an dieser Stelle darauf, dass ein neuerer spanischer Band von Juan Manuel de Prada mit dem schönen Titel Coños, unverkennbar ein kommerzielles Remake nach einer Überbietungsstrategie des in unserer Vorlesung behandelten Avantgardetextes Senos 
von Ramón Gómez de la Serna, ${ }^{2}$ einen Prosatext auch Georges Bataille widmet. Sein Autor, mit allen kommerziellen Wassern der postfranquistischen spanischen „Movida“ gewaschen, erwies durch eine kleine Hommage dieser Eingangsszene und deren Autor Georges Bataille eine literarische Ehrung, welche freilich ein bloßes Anzitieren einer in der Literaturgeschichte berüchtigten Szene nicht überschreitet.

Unsere Novelle beginnt also mit einer erotischen Entdeckungs- und Erkenntnisreise zweier Minderjähriger, die den Ausgangspunkt für die Erfahrungsgeschichte des Erzählers bildet. Die einzelnen Elemente und Handlungsteile der Histoire de l'Eil, auf welche ich gleich zurückkomme, werden in einem nachgestellten kurzen Kapitel in einen Zusammenhang mit autobiographisch dargestellten Erlebnissen des Ich in Verbindung gebracht. Trotz des verwendeten Pseudonyms und der offenkundig begründeten Angst des Verfassers vor der französischen Zensur bildet erneut das Leben und mehr noch das Erlebte das eigentliche Zentrum der Erzählung. Diese Dimension eines autobiographisch fundierten Lebenswissens verleiht dem Erzählten seine eigentliche Bedeutung jenseits rein fiktionaler Gestaltungsformen.

In den nachgestellten „Réminiscences“ werden einzelne Episoden als Auslöser für die in der Novelle dargestellten Handlungen in einer Weise verwendet, die für uns sehr aufschlussreich ist und die keineswegs durchgängig zu beobachtende Ästhetik des Bruches bei Bataille mit dem Aspekt eines sexuellen Tabubruches verbindet:

Ich bin als Sohn eines syphilitischen (schwindsüchtigen) Vaters geboren. Er wurde blind (er war dies schon bei meiner Zeugung), und als ich zwei oder drei Jahre alt war, lähmte ihn diese Krankheit auch noch. Als kleines Kind bewunderte ich diesen Vater. Lähmung und Blindheit hatten freilich Folgen, zum Beispiel: Er konnte nicht wie wir zum Pissen auf die Toilette gehen; er pisste aus seinem Sessel, er hatte dafür ein Gefäß. Er pisste vor meinen Augen, unter einer Decke, die er als Blinder falsch hinlegte. [...]

Meine Mutter verschwand eines Tages, indem sie einen Augenblick nutzte, als ich ihr den Rücken zudrehte. Wir haben sie lange gesucht; mein Bruder fand sie rechtzeitig wieder, sie hatte sich im Speicher zu erhängen versucht. So kehrte sie immerhin ins Leben zurück. Sie verschwand ein weiteres Mal: Ich musste sie endlos lange entlang des Baches suchen, in welchem sie sich hätte ertränken können. [...] Sie war selbst wieder aus dem eisigen Wasser des Baches gestiegen (denn es war mitten im Winter), denn das Wasser war an diesem Ort nicht tief genug, um sich zu ertränken.

2 Vgl. hierzu ausführlicher Ette, Ottmar: Mit Haut und Haar? Körperliches und Leibhaftiges bei Ramón Gómez de la Serna, Luisa Futoransky und Juan Manuel de Prada. In: Romanistische Zeitschrift für Literaturgeschichte / Cahiers d'Histoire des Littératures Romanes (Heidelberg) XXV, 3-4 (2001), S. 429-465. 
An diesen Erinnerungen bleibe ich gewöhnlich nicht hängen. Nach langen Jahren haben sie die Macht verloren, mich zu erreichen: Die Zeit hat sie neutralisiert. Sie konnten das Leben nur verformt, fast unkenntlich wiederfinden und hatten im Verlauf ihrer Verformung einen obszönen Sinn angenommen. ${ }^{3}$

In dieser autobiographischen Rückschau des realen Autors Georges Bataille wird deutlich, wie sehr in die fiktionale Geschichte, in die Novelle, autobiographische Elemente Eingang gefunden haben. Nicht zu Unrecht könnte man darauf verweisen, dass wir in diesen autobiographischen Passagen eine Vielzahl von Textelementen finden, die Bataille einer dreifachen, von der Psychoanalyse Sigmund Freuds analysierten Arbeit unterworfen hat, nämlich jener von Verschiebung, Verdichtung und Verdrängung. In diesen Verfahren aus Freuds Traumdeutung erkennen wir leicht jene literarischen Verfahren, welche in diese Novelle eingeflossen sind.

In den bereits oben angeführten Passagen wird erkennbar, wie stark die Erfahrungen des jungen Bataille körperlicher Natur sind, wie sehr ihm der Körper seines blinden und fast völlig hilflosen, zu guter Letzt dem Wahnsinn verfallenen Vaters gegenwärtig ist, und wie sehr die körperlichen Funktionen notwendig in dieses literarische Schreiben Eingang fanden. Zugleich bemerken wir einen Willen, alles zu sagen, alles zum Ausdruck zu bringen, nichts zu verbergen oder zu beschönigen. Diese „volonté de tout dire“ ist ohne jeden Zweifel das Charakteristikum des Schreibens von Georges Bataille. Eine Tatsache, die in keiner Weise weit entfernt ist von eben derselben, freilich zeithistorisch bedingt sich anders äußernden autobiographischen „écriture“ jenes Schöpfers der modernen Autobiographie, der mit dem Buch in der Hand einstmals vor seinen Schöpfer durchaus in der Position des Anklagenden - trat: Jean-Jacques Rousseau. ${ }^{4}$ Bataille befindet sich in diesem Schreiben folglich in guter Gesellschaft und in einer langen literarisch-philosophischen Tradition. Die Radikalität seines autobiographischen Schreibaktes ist jedoch prononcierter.

Die durchaus vorromantische Tradition Rousseau'scher Provenienz beinhaltet bei Bataille noch zumindest zwei weitere Elemente. Zum einen ist sein Schreiben geknüpft an das Brechen gesellschaftlicher Tabus und verlangt nach einer grundlegenden Revolution, die noch weit über das hinausgehen sollte, was die Surrealisten - die Bataille stets als simple idealistische Idioten abtat - gefordert hatten. Und zum anderen war es ein Zeitgenosse Rousseaus, der Marquis

3 Bataille, Georges: Réminiscences. In (ders.): Euvres complètes I, S. 607 f.

4 Vgl. das Rousseaus Autobiographie gewidmete Kapitel in Ette, Ottmar: LiebeLesen. Potsdamer Vorlesungen über ein großes Gefühl und dessen Aneignung. Berlin - Boston: Verlag Walter de Gruyter 2020. 
de Sade, der ihn in einer interessanten Konstellation dieser beiden Männer des 18. Jahrhunderts zu einem Schreiben führte, in welchem sich das Revolutionäre mit dem Autobiographischen und das Körperliche mit dem Psychologischen (und vielleicht auch mit dem Psychiatrischen) verbindet. Gleichzeitig sehen wir, dass gerade bezüglich der letztgenannten Dimension die Distanz zwischen Breton und Bataille gar nicht so riesig und unüberwindlich war.

Ziel des Schreibens von Bataille war dabei die literarische Erfassung des Obszönen, mithin dessen, was in der Literatur eigentlich nicht mehr ,erlaubt“ ist. So wie seine Schriften sich ständig zwischen Roman, Abhandlung, Pamphlet oder Essay hin und her bewegten, so akzeptierte er auch auf anderen Gebieten keine Grenzen. Grenzen boten für ihn stets den Grund für Grenzübertretungen. Der Anspruch des Schreibens von Bataille war immer total und absolut. Er wollte eine Art Nacktheit zum literarischen Ausdruck bringen, die keineswegs musealen und voyeuristischen Charakter besitzt, sondern grundsätzlich auf das in einer bestimmten Epoche nicht mehr Darstellbare abzielt. Sein Schreiben stand stets im Zeichen der „Verausgabung“, eines wichtigen Theorems der Philosophie und Literaturtheorie dieses französischen Schriftstellers.

So blieb das Obszöne kein Betriebsunfall, sondern ein gewolltes Ziel seines grenzüberschreitenden Schreibens. Vor diesem Hintergrund verstehen wir besser, warum es bei Bataille gegenüber André Breton eine grundlegende Inkompatibilität gab, warum Bataille dem ,Chef' des französischen Surrealismus vorwerfen konnte, nicht nur ein Diktator im intellektuellen Feld zu sein, sondern zugleich auch ein Moralist, Purist und Idealist, der keineswegs eine wirklich fundamentale Revolution vorhabe. Innerhalb eines solchen Kontexts wird auch verständlich, warum Bataille mit seiner 1929 gegründeten Zeitschrift Documents zu einem Hort für die ausgestoßenen, die radikaleren, die dissidenten Surrealisten werden konnte und warum seine Zeitschrift zu einer wahren „machine de guerre“ gegen Bretons erfolgreiche Truppe werden musste. Es wird klar, warum später der Surrealismus Bretons so sehr auch auf dem öffentlichen nationalen Parkett glänzen konnte, während Bataille stets - wie Mario Vargas Llosa gleich zu Beginn seines schönen Essays über Bataille schrieb - ein Schriftsteller für Minderheiten blieb und wohl auch immer bleiben werde. ${ }^{5}$ Daran hat sich auch bis heute nichts geändert.

Georges Bataille, ein Marginalisierter also, gar ein Ausgestoßener? Das ist gleichzeitig richtig und verkehrt. Denn diese Minderheitenrolle von Bataille war

5 Vgl. Vargas Llosa, Mario: Sobre Bataille: respuesta a García Ponce. In (ders.): Contra viento y marea II (1972-1983). Barcelona: Seix Barral 1983, S. 131-134 (der Artikel stammt aus dem Jahre 1979). 
sehr einflussreich, insofern er mit seinem Denken in der Tat die Neo-Avantgarde der Philosophie grundlegend beeinflusste, die sich im Zeichen des Poststrukturalismus in der zweiten Hälfte des 20. Jahrhunderts aufmachte, mit den alten Grenzen einer idealistischen wie materialistischen Philosophie endgültig Schluss zu machen. So hat er herausragende Denker wie Michel Foucault, Roland Barthes oder Gilles Deleuze und Jacques Derrida sowie viele andere mehr außerhalb der Grenzen Frankreichs fundamental beeinflusst und entscheidend mitgeprägt.

Es gibt im Verlauf von Histoire de l'Fil eine Reihe von Szenen, die ich Ihnen im Rahmen unserer Vorlesung lieber nicht vorstellen möchte. Sie können sich ja jederzeit diesen Band, den ich Ihrer aufmerksamen Lektüre anempfehle, selbst vornehmen! Sie haben faktisch alle erotischen Stellungen menschlicher Körper literarisch vor sich: in Form der Masturbation, des Geschlechtsverkehrs, des Analverkehrs, des Beischlafs mit Tieren, der Verbindung mit einer langsamen Strangulierung, einer sadistischen wie masochistischen Zurschaustellung sexueller Erregung und Befriedigung und vieles mehr ... Es handelt sich um eine Fülle an Szenen, die gerade im Rahmen einer Vorlesung nicht jedermanns und ,jederfraus Sache sind. Ähnlich wie beim Marquis de Sade hat man in den sechziger Jahren zunehmend versucht, diese Szenen als eine Art Sprache zu verstehen, wobei die einzelnen menschlichen Körper gleichsam eine Grammatik der Figuren aufführen, welche abstrakt, von den Körpern also abgezogen, zu Kunstwerken werden. Dies hat sicherlich dazu beigetragen, neben dem Marquis de Sade auch Georges Bataille nicht allein in seinen theoretischen Texten schulfähig und hoffähig zu machen. Mann und Frau sollten sich freilich dem schonungslosen Tabubruch Bataille'schen Schreibens unmittelbar und nicht abstrahierend aussetzen, um die ganze Tragweite einer derartigen „écriture“ zu erfassen.

Ich möchte Ihnen zum Abschluss unserer Beschäftigung mit Georges Bataille nicht das einigermaßen schreckliche Ende vorführen, das Simone, der Ich-Erzähler und ein voyeuristischer englischer Lord in Sevilla erleben. Bataille ließ in diese Szenerie seine Erlebnisse in der Stierkampfarena zu Madrid einfließen, wo er den Tod des jungen Toreros mitansehen musste. In Batailles Fiktion begehen diese drei Protagonisten einen langsamen Ritual- und Sexualmord an einem Priester, der nach allen Regeln der Kunst zunächst verführt und dann martyrisiert wird. Tötete Bataille in dieser Szene den Priester in sich selbst, der er wohl immer noch war? Ich will Ihnen auch nicht davon berichten, dass die Dreierbande dann schließlich aus Spanien fliehen kann, ohne erwischt zu werden, und so auch in Zukunft ihr düsteres Treiben fortsetzen kann. Denn am Ende kauft Lord Edward eine Yacht, mit Hilfe derer man mit einer schwarzen Besatzung dann Kurs auf neue Tabubrechungen nimmt.

Nein, ich möchte mit Ihnen auch nicht jene spezifisch spanische Dimension erforschen, in welcher aus französischem Blickwinkel Spanien immer für eine 
unterdrückte, dann aber leicht explosive Sexualität steht, die stets unter einem seltsam fixierten Himmel, in praller Sonne und mit voller Gewalt, vonstattengeht! Aber ich gebe Ihnen doch einen gewissen Vorgeschmack hierauf in einer Szene, die natürlich ,typisch spanisch“ ist und sich in der Arena von Madrid zuträgt, kurz bevor die drei verbrecherischen Tabubrecher in Richtung Sevilla weiterfahren. Dabei ist die Stierkampfarena natürlich jener Ort, an dem sich Gewalt und Tod, Lust am Tod und Lust an der Liebe, Brutalität und eruptive Sexualität miteinander verbinden und vermengen.

Einen ersten Höhepunkt bildet in diesem Handlungsstrang jene Szene, die Georges Bataille nach eigenen Angaben direkt erlebt haben will, nämlich das schreckliche Ende eines jungen spanischen Torero vor den Augen einer in jeglicher Hinsicht erregten Menschenmenge. Wichtig zum Verständnis der Szenerie ist, dass sich nach dem Tod des ersten wilden Stiers Simone durch Lord Edward die Stierhoden auf einem Teller - und Sie verstehen die Wiederholungsmechanismen im Text sofort! - hatte bringen lassen, nicht etwa um sie aufzuessen (was man in Spanien übrigens bis heute tut), sondern um sich wie in der Eingangsszene auf sie zu setzen und damit das eigene Geschlecht zu befriedigen. Nun aber kommt es in der Folge zu einer seltsamen Koinzidenz. Denn diese runden Formen kehren wieder in den Augen jenes begehrten und anziehenden spanischen Stierkämpfers namens Granero, der vor aller Augen ein grausames Ende findet. Die literarische Szene ist übrigens datiert auf den 7. Mai 1922 und lebt ganz in surrealistischer Manier von den vielen Koinzidenzen, Déjà-vus und Überschneidungen, welche der Text mit seinen Freud'schen Überdeterminierungen und Traumsymboliken produziert und inszeniert:

\footnotetext{
Was folgte, fand ohne Übergang statt und sogar augenscheinlich ohne jede Verbindung, nicht dass die Dinge nicht miteinander verbunden gewesen wären, sondern weil ich sie wie ein Abwesender sah. Ich sah zu meinem Grauen binnen weniger Augenblicke Simone in eine der Hodengloben beißen, Granero vorrücken, wie er dem Stier das rote Tuch zeigte; dann sah ich Simone, der das Blut in den Kopf gestiegen war, in einem Augenblick von schwerer Obszönität ihre Scheide enthüllen, wohin die andere Stierhode verschwand; dann sah ich Granero umgestoßen, unter der Balustrade an die Wand gedrückt, dann stießen auf dieser Balustrade die Stierhörner dreimal zu: Eines der Hörner stieß in das rechte Auge und den Kopf. Der entsetzte Aufschrei der Arena fiel mit dem Höhepunkt von Simone zusammen. Vom Steinpflaster erhoben, schwankte sie und fiel, die Sonne machte sie blind, sie blutete aus der Nase. Einige Männer stürzten herbei, packten Granero.

Die Menge in der Arena war komplett aufgesprungen. Das rechte Auge des Kadavers hing herunter. ${ }^{6}$
}

6 Bataille, Georges: Histoire de l'Eil, $596 \mathrm{f}$. 
Sie haben nun einen plastischen Eindruck von der Gewalt der Sprache, deren sich Georges Bataille bedient. Es ist eine Sprache, die in ihrer ungeglätteten Brutalität alles mit sich fortreißt und keinerlei Nuancierung duldet: Atemlos werden die einzelnen Ereignisse zum Teil asyndetisch hintereinander geschaltet, ohne doch logisch und kausal miteinander verbunden zu sein. In dieser Szene, in deren Mittelpunkt die augenförmigen, eiförmigen Gegenstände stehen, ist der Ich-Erzähler mit Hilfe seiner Augen - beachten Sie das Insistieren auf dieser Augenzeugenschaft von Beginn der Passage an mit ihren Rekurrenzen des Lexems „voir“ - der „témoin“ einer Überlagerung geradezu tagtraumartig erscheinender Bildsequenzen, die sich a-kausal überlagern.

Die blutig-erotische Dimension überschneidet sich in dieser Szenerie mit einer Gewalttätigkeit, die deutlich an den surrealistischen Film Le chien andalou von Buñuel und Dalí erinnert: Nicht umsonst hatte auch dort - Sie erinnern sich bestimmt! - das Auge in jener Szene seines Aufschneidens mit Hilfe eines Rasiermessers im Zentrum gestanden. Das zuerst von einer Wolke und dann, in einer bildhaften Überschneidung, von einem Rasiermesser aufgeschnittene Auge koinzidiert mit der brutalen Todesart des Torero, dessen Kopf und Augenhöhle von einem Stierhorn durchbohrt wird.

Doch dieser grässliche Tod des Toreros fällt im Text Batailles mit dem Höhepunkt Simones, mit der gleich zweifachen Einverleibung der Stierhoden, zusammen. Simones Lust, die zuvor bereits durch den Erzähler zwar brutal, aber offenkundig nicht ausreichend befriedigt worden war, wird so bis zum Äußersten gesteigert und koinzidiert mit dem Tod des Stierkämpfers, wobei sich zwischen beiden eigentlich unverbundenen Serien geradezu ein kausaler Nexus zu etablieren scheint. Dieser Nexus entsteht nicht nur durch die Koinzidenz in zeitlicher und räumlicher Hinsicht, sondern eben auch durch die Parallelität der ei- oder augenförmigen Gegenstände, die ja auch im Zentrum des Titels dieser Novelle stehen. Wenden wir uns daher kurz diesen plastisch geschilderten Formen von Auge, Stierhoden und ,globes“ zu.

Denn das Auge steht für den menschlichen Gesichtssinn, das Sehen, und ist zugleich ein Teil des menschlichen Gehirns, mit dem es durch die Augenhöhlen direkt verbunden ist. Das Auge ist in der Tradition der europäischen Mystik das Fenster zur Seele, steht aber zugleich auch für die Sonne ein, deren runde Form es besitzt. In einer langen literarisch-philosophischen Tradition wurde die Strahlkraft des Auges mit jener der Sonne in eins gesetzt, stellte man sich doch vor, dass der Mensch allein auf Grund der Strahlkraft seiner Augen sehen könne. Erlahmte oder erlosch diese, so wurde der Mensch - wie der Vater von Georges Bataille blind. Seit der kleine Junge die Blindheit seines Vaters hautnah erlebte, war das Auge ein traumatisch immer wiederkehrendes Objekt in seinem Denken wie in seinem späteren Schreiben. 
Gleichzeitig aber stand der Augapfel mit dem Erdapfel in Beziehung, stand also der Globus des Auges für den der Erde ein und umfasste dergestalt eine ganze Welt, einen ganzen Kosmos, der sich in allen Teilen dieses Universums, beispielsweise in den Sternen, wie am menschlichen Körper, etwa in Form der Hoden des Mannes, figural wiederfinden ließ. Auf diese Weise steht diese Kugel- oder Eiform als Pars pro toto für ein ganzes Universum, für den ganzen Menschen, den ganzen Körper ein, der in seiner Kraft und Stärke evoziert wird. Daher rührt auch die nach heutigem medizinischem Wissen irrige Vorstellung, der zufolge die männliche Kraft in diesem Organ lokalisiert sei.

Auf eben dieser Ebene entsprechen sich in der Histoire de l'Fil die Geschlechtsorgane von Mann und Frau. So wird ganz am Ende die Scheide Simones für den Ich-Erzähler jene Form eines menschlichen Auges annehmen, in dem dann plötzlich für ihn das blaue Auge der früher bei derartigen Spielen ums Leben gekommenen Marcelle sichtbar wird. Ein Auge, ein Globus, verbirgt viele Augen, viele Globen. Zweifellos: Histoire de l'Eil ist ein Grenztext, der die von den Surrealisten verkündete Revolution noch wesentlich grundsätzlicher und offensiver aufnimmt, ja auskostet. Und diese eklatante Differenz wird gerade anhand der fast gleichzeitig entstandenen Texte Nadja und Histoire de l'Eil im wahrsten Sinne des Wortes augenfällig. Sie bildet durchaus den Grund dafür, Georges Bataille im Kontext des Surrealismus zu behandeln, auch wenn er selbst dieser Gruppe und Truppe der historischen Avantgarde nur sehr kurzzeitig nahe gestanden und sie lange Zeit bekämpft hatte.

Vehement und offensiv nahm bekanntlich Breton nicht nur in seinem Second Manifeste du Surréalisme den Kampf gegen Bataille auf. Es macht im Rahmen unserer Vorlesung keinen Sinn, die gegenseitigen Anfeindungen der beiden in aller Genauigkeit zu verfolgen. Gewiss war die Gegenattacke Batailles und seiner Freunde rund um die Zeitschrift Documents sehr schön, mit Hilfe etwa ihres wohlgezielten Pamphlets Un Cadavre Schaden bei den Surrealisten anzurichten, spielte dieser Text doch schon im Titel auf Bretons und Aragons herbe Attacken gegen Anatole France zehn Jahre zuvor an. Wir können auch nicht eingehen auf die zeitweilige, aber eher kurzzeitige Zusammenarbeit rund um die Gruppe „Contre-Attaque“, die recht ergebnislos unter Führung Batailles versuchte, eine Einheit der - wie es hieß - „revolutionären Intellektuellen“ gegen den überall aufkeimenden Faschismus herzustellen. Wichtig ist mir vor allem, dass Sie die strukturelle Verwandtschaft zwischen diesen beiden großen französischen Vertretern avantgardistischer Positionen im Magnetfeld des Surrealismus verstehen!

Aufschlussreich ist in diesem Zusammenhang bereits, was Breton Bataille 1930 in seinem Second Manifeste vorzuwerfen hatte. So möchte ich unsere Auseinandersetzung mit dem Surrealismus in Bezug auf die französischen Primärtexte mit einem Auszug aus einem avantgardistischen Manifest beenden, wie es 
sich für eine abschließende Auseinandersetzung mit der Epoche der historischen Avantgarden gehört. Dies bedeutet freilich nicht, dass in unserer Vorlesung André Breton - wie in so vielen anderen Geschichten der Avantgarden und des Surrealismus - das letzte Wort behalten soll:

\begin{abstract}
Herr Bataille interessiert mich ausschließlich in dem Maße, in dem er sich schmeichelt, sich gegen die harte Disziplin des Geistes aufzulehnen, der wir offenbar alles unterzuordnen suchen - und wir erblicken keinen Nachteil darin, dass dafür hauptsächlich Hegel verantwortlich gemacht wird -, eine Disziplin, der es nicht einmal gelingt, als eher feige zu erscheinen, tendiert sie doch dazu, jene des Nicht-Geistes zu sein (und genau darauf wartet im Übrigen Hegel). Herr Bataille verweist öffentlich darauf, nur das auf der Welt in Betracht ziehen zu wollen, was das Gemeinste, das Entmutigendste und das Korrumpierteste ist [...]. Es amüsiert mich im Übrigen zu denken, das man aus dem Surrealismus nicht herauskommen kann, ohne über Herrn Bataille zu stolpern, insofern es wahr ist, dass die Abscheu vor der Strenge sich nur in eine neuerliche Unterwerfung unter die Strenge übersetzen lässt. Mit Herrn Bataille, daran ist nichts neu, sehen wir eine offensive Rückkehr des alten antidialektischen Materialismus kommen, der dieses Mal versucht, sich kostenlos einen Weg durch Freud zu bahnen. ${ }^{7}$
\end{abstract}

Die Attacken André Bretons zielen zunächst vor dem Hintergrund des eigenen Organisationsgrades gegen eine sich um Bataille scharende Gruppe, welche bei weitem nicht die strenge Organisation der „surréalistes“ erreicht hatte oder besser erreichen wollte. Die Positionskämpfe der französischen Surrealisten im literarisch-künstlerischen Feld hatten bereits 1929 zu einer direkten Kampfansage an die damals rivalisierende Zeitschrift Le Grand Jeu geführt, wobei die Surrealisten unter der Führung Bretons eine Gruppendisziplin erreichten, welche sich auf zwei Ebenen klar abzeichnete. Zum einen auf der Status-Ebene ihrer künstlerischgesellschaftlichen Zusammengehörigkeit als surrealistische Künstler und Schriftsteller, zum anderen auf Ebene ihrer Zugehörigkeit zur Kommunistischen Partei Frankreichs. All dies unterschied die Surrealisten deutlich von jener außerhalb derartiger Strukturen stehenden Gruppe um die von Bataille geführte Zeitschrift Documents, der sich - wie Breton auch im Manifest festhielt - unter anderem die abtrünnigen Surrealisten Desnos, Leiris, Limbour, Masson oder Vitrac angeschlossen hatten. Dies waren, dessen war sich André Breton sehr wohl bewusst, herausragende Autoren und Künstler, die nicht mehr zum Establishment des Surrealismus gehörten, aber gleichwohl Karriere machten und bis heute wichtige Vertreter aus dem Umfeld des Surrealismus darstellen.

Kein Zweifel kann daran bestehen, dass Breton in Bataille und der Zeitschrift Documents zum damaligen Zeitpunkt die gefährlichsten Gegner seines eigenen

7 Breton, André: Les manifestes du surréalisme. Paris: Le Sagittaire 1955, S. 90 f. 
,institutionalisierten' Surrealismus sah. Daher auch der Kampf um Hegel, den sowohl die Surrealisten als auch Bataille, der Hegel mit den Augen und als Schüler von Kojève gelesen hatte, für sich gleichermaßen reklamierten. Der zweite Vorwurf Bretons zielt auf die Inhalte literarischer Darstellungen Batailles ab, und man darf sehr wohl festhalten, dass die Argumente Bretons alles andere als revolutionär waren: Sie sind im Grunde konventionell, kleinbürgerlich und bis ins Detail jenen vergleichbar, welche ein halbes Jahrhundert zuvor der psychologische Roman gegen den siegreich vordringenden Naturalismus vorgebracht hatte. Georges Bataille sollte also marginalisiert werden, weil er sich mit dem Übelriechenden der Gesellschaft einließ.

Andererseits war klar, dass in der Überkreuzung beider Argumentationsebenen gegen den Vorwurf des Idealismus, den Bataille und andere dem Surrealismus machten, nun der Vorwurf des Materialismus errichtet wurde, der alles undialektisch auf bloße Materie reduziert wissen wolle. Die ausgetauschten Argumente und die damit jeweils verbundenen Vorwürfe waren schlicht vorhersehbar und keineswegs originell. In Bretons Angriffslinie sehen wir deutlich die Attacke gegen die Betonung des Materiellen im Sinne des Physisch-Körperlichen, welche als Zurschaustellung des Gemeinen, Unappetitlichen verunglimpft wird. Was damit wirklich gemeint ist, wissen wir nach unserer Lektüre von Histoire de l'Eil freilich besser! Dass man gerade diese Materialität aber auch entmaterialisieren und einer gegenüber der Lesart André Bretons ganz anderen Lektüre unterziehen kann, soll unser letzter Schritt in diesem Kapitel zeigen.

In seinem als Hommage an Georges Bataille in der Zeitschrift Critique 1963 erstmals veröffentlichten Text La métaphore de l'œil ${ }^{8}$ betonte der französische Kulturkritiker und Schriftsteller Roland Barthes von Beginn an, dass Bataille in seiner Histoire de l'Eil nicht die Geschichte des Erzählers, Simones oder Marcelles geschrieben, sondern vielmehr dargestellt habe, wie ein Objekt, ein Gegenstand, eine eigene Geschichte haben könnte. Damit führte Barthes gleich vom Anfang seines Essays an eine gewisse Entkörperlichung und Abstraktion ein, welche Batailles Novelle sicherlich absichtsvoll entschärft. Bataille habe die Migrationen des Auges zu anderen Objekten dargestellt und habe damit nicht mehr den Gegenstand einer Imagination, sondern die Imagination selbst beschrieben.

Diese als abstrakt zu bezeichnende Sichtweise eröffnete in der Tat eine spannende Untersuchungsperspektive, die freilich zugleich die erotische Dimension zwar nicht ausklammerte, aber doch in den Hintergrund schob, so als stünde sie nicht vom Vorwort Batailles an im Mittelpunkt der Novelle. Aus zum dama-

8 Vgl. Barthes, Roland: La métaphore de l'œil. In (ders.): Euvres critiques. Edition établie et présentée par Eric Marty. Bd. I. Paris: Editions du Seuil 1993, S. 1346-1351. 
ligen Zeitpunkt strukturalistisch-linguistischer Sicht sah Barthes die Variationen eines gewissen Terms, welcher durch bestimmte Substitutionsmechanismen ersetzt werden könne: ein gewiss überraschender linguistischer Vorgang, mit dem Barthes einen neuen Lektüremodus von Bataille eröffnete. Denn alles ist in gewisser Weise „globuleux“, zugleich aber auch „dissemblable“.

So seien wie in einem Paradigma von Georges Bataille die Substitute des Auges konsequent durchdekliniert worden. Das zeige sich schon in der ersten Variation zwischen Auge und Ei, die im Französischen ja als „œil“ und „œuf“ ja auch in großer lautlicher Nähe zueinander stehen. Wir hatten die große Bedeutung derartiger Verknüpfungen für Bataille bereits am Beispiel „assiette“ (welche in der späteren Szene der Stierkampfarena wiederkehrt) und „assoir“ gesehen. Parallel zu dieser ersten metaphorischen Kette zwischen „œil“ und „œuf“ verlaufe eine zweite, jene der damit jeweils verbundenen Flüssigkeiten (also von der Milch über die Tränen bis zum Sperma), wobei die eine Kette stets die andere aufrufe. Signifikant ist hierbei auch, dass die Sonne mit ihrer physischen Gewalt anders als in den germanischen ist in den romanischen Ländern ,die liebe Sonne‘ nicht weiblich, sondern hart, gewalttätig und männlich - zugleich trocken und ei- oder augenförmig ist, sich also sehr wohl in diese Kette von „objets“ eingliedert, welche eine komplexe und vieldeutige Isotopie bilden.

Innerhalb dieser hochgradig vernetzten Isotopie gibt es aber kein zentrales Signifikat, auf das alles zurückgeführt werden könnte, schon gar nicht das Erotische oder Sexuelle, das - so Barthes - hier keineswegs im Zentrum stehe, so dass sich alles von ihm ableiten lasse. In gewisser Weise herrsche das Auge aber unverkennbar vor, denn es steht im Titel der Bataille'schen Novelle - und schließlich war Batailles Vater, wie es direkt in der von uns untersuchten Reminiscence angeführt wurde, blind. Bei ihm wurde das Weiße im Auge sichtbar, wenn er vor dem Kind urinierte. Also könne es sich - so Barthes weiter - letztlich höchstens um eine Verbindung zwischen dem Okulären und dem Genitalen handeln.

Ich möchte mich an dieser Stelle nicht auf die Abgrenzung zwischen Bataille und dem Marquis de Sade konzentrieren, die Barthes gegen Ende seines Essays sehr hellsichtig durchführte, sondern auf einen Kern seiner strukturalistischen Analyse. Lassen Sie mich hier nur ein kleines Beispielszitat einfügen:

Diese Kunst ist keineswegs eine Spielerei, insofern sie sich, wie es scheint, mit dem Erotisierenden selbst, zumindest mit dem von Bataille, vermischt. Gewiss kann man sich für das Erotisierende andere als linguistische Definitionen vorstellen (und Bataille selbst hat dies gezeigt). Aber wenn man als Metonymie jene Übersetzung von Sinn bezeichnet, die sich von einer Kette auf eine andere abspielt, auf verschiedene Ebenen der Metapher (das Auge, an dem wie an einer Brust gesaugt wird, mein Auge zwischen ihren Lippen trinken), dann wird man ohne Zweifel einräumen, dass das Erotisierende bei Bataille im wesentlichen metonymischer Natur ist. Da die poetische Technik hier darin besteht, die üblichen Kontiguitäten 
von Objekten abzustreifen, um dafür neue Treffen zu substituieren, welche freilich durch die Persistenz eines einzigen Themas im Inneren jeder Metapher begrenzt sind, kommt es zu einer Art allgemeiner Ansteckung von Qualitäten und Handlungen [...]. ${ }^{9}$

An dieser Stelle wird deutlich, wie die Neo-Avantgarden (für die Roland Barthes hier stellvertretend stehen mag) die alten, historischen Avantgarden ihrerseits dazu benutzen, um bislang unbedachte und nicht mehr zentrierte Sinnsysteme zuerst einzuführen und gleichzeitig noch weiterzudenken, dabei aber kontinuierlich in ihrer Ausrichtung und Zielstellung bewusst $z u$ verstellen. ${ }^{10}$ Denn durch diese gezielte Verstellung verschwindet die ganze Wucht dieses Textes mit seinen immens schockierenden Bildern in einem linguistisch-strukturalistischen Schema, das nur noch Modi der Kommunikation von Metapher und Metonymie, von Similarität und Kontiguität, von Austausch und Überkreuzung kennt. Dieses linguistisch inspirierte Schema aber ist an keine physische, hautnahe Körperlichkeit mehr rückgebunden: Von eben dieser körperlichen Leibhaftigkeit ist es bewusst abgezogen, wirkt folglich abstrakt und nicht mehr provozierend oder schockierend.

Lassen Sie mich mit diesen Reflexionen zu einer Schlussüberlegung dieser Vorlesung vorstoßen, die mit Georges Bataille sicherlich an einen Grenz-Text der Moderne und zugleich an eine Grenze gekommen ist, welche in der Folge zumindest von den historischen Avantgarden eigentlich kaum noch überboten werden konnte! Wir befinden uns an einem Punkt der Vorlesung, an welchem sich das Scharnier zwischen Moderne und Postmoderne vorzüglich $\mathrm{zu}$ bewegen scheint. Genau an diesem Bewegungsscharnier der Moderne aber werden wir uns nun in den folgenden Sitzungen eine ganze Weile aufhalten.

Doch bevor wir uns den diesen Teil abschließenden Überlegungen zuwenden, sei nicht vergessen, dass sich aus jener dargelegten Kombinatorik von Lust und Tod, von Eros und Thanatos, von Rituellem und Textuellem das besondere Interesse Georges Batailles auch und gerade an kulturellen Praktiken erklärt, die sich außerhalb Europas ansiedeln. Er ist darin ein getreuer Weggenosse eines anderen ,abtrünnigen' Surrealisten, Antonin Artaud, der sich (wie im Übrigen auch Breton selbst) Mexiko zuwandte und dort nach bewusstseinserweiternden, die abendländische Logik radikal durchbrechenden Kulturelementen und Stoffen suchte.

9 Barthes, Roland: La métaphore de l'œil, S. 1350.

10 Vgl. hierzu Ette, Ottmar: Der Schriftsteller als Sprachendieb. Versuch über Roland Barthes und die Philosophie. In: Nagl, Ludwig / Silverman, Hugh J. (Hg.): Textualität der Philosophie: Philosophie und Literatur. Wien - München: R. Oldenbourg Verlag 1994, S. 161-189. 
In den Riten und Regeln des haitianischen Voodoo, mehr aber noch in den Opferriten der Azteken erblickte Georges Bataille durchaus jene Kombinatorik von Tod und Lust, Anstauung und Verausgabung, die ihn hemmungslos interessierte: jene Art der „dépense“, die er in seinen eigenen Figurenkonstellationen mehrfach $\mathrm{zu}$ entwerfen, $\mathrm{zu}$ analysieren und vor allem theoretisch $\mathrm{zu}$ verarbeiten versuchte. Der Surrealismus - und gerade auch der dissidente Surrealismus - öffnete sich in sehr grundlegender Weise gegenüber Kulturen und Kulten, weniger aber den Literaturen Außereuropas, auch wenn er diese Öffnung gegenüber dem Transkulturellen - der Begriff stammt just aus dem Jahr 1940 und aus ebendiesem geographischen Raum der Karibik - noch in einer fundamental abendländischen und eurozentrischen Weise vollzog.

Mit dieser Öffnung auf das Transkulturelle aber inaugurierte und schuf er jene Möglichkeiten, die uns heute noch in Bann halten. So beginnen die historischen Avantgarden, jene Beziehung zwischen den Welten des Abendlandes und der nicht-okzidentalen Welt auf eine radikal andere Weise im Zeichen einer transkulturellen Transformation zu denken, insofern diese Relation nicht mehr als Bruch, sondern als Kontiguität imaginiert und vorgestellt werden kann. Erneut geht es bei diesem Erleben anderer kultureller Erfahrungsweisen um eine Versöhnung von Kunst und Leben, von Literatur und Leben, wie wir sie in künftigen Sitzungen unserer Vorlesung zu besprechen haben werden. Auch dies bestätigt einmal mehr unsere These, dass das Lebenswissen der historischen Avantgarden auch in der zweiten Hälfte des 20. Jahrhunderts und im Übrigen bis heute höchst präsent und aktuell ist.

Mit anderen Worten: Es ist die Suche nach einer anderen Logik, die nicht mehr die eines Bruches, sondern einer weiteren und erweiterten, viellogischen Logik ist, die sich uns öffnet als eine ebenso radikale wie fundamentale Möglichkeit, aus der eigenen Ästhetik des Bruches und der okzidentalen Zielstrebigkeit auszubrechen. Max Aubs Jussep Torres Campalans wird uns später wichtige Erkenntnisse und Einsichten in diese Komplexität der Beziehungen liefern. Wieviel Romantik auf der anderen historischen Seite noch immer in den historischen Avantgarden steckt, mag man zweifellos an der Idealisierung dieser außereuropäischen Welten ermessen, denen sich im Gefolge dann Anthropologen und Ethnologen wie Michel Leiris widmeten. Doch der Surrealismus versuchte zum ersten Mal radikal, dieses Denken in irgendeiner Weise auf unsere eigene Gegenwart, unsere eigene europäische Welt zu beziehen. Er öffnete damit ein Tor, das in unserer gegenwärtig wieder kleiner werdenden europäischen Welt noch längst nicht völlig aufgestoßen ist. Doch wollen wir in unserer Vorlesung auch weiterhin versuchen, für diese Öffnung eine Lanze zu brechen. 